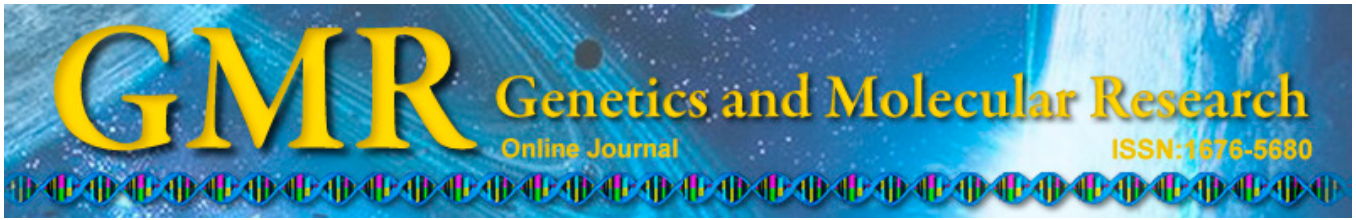

\title{
Effect of flavonoid compounds extracted from Iris species in prevention of carbon tetrachloride-induced liver fibrosis in rats
}

\author{
Y.L. Wang, H.Y. Lv and Q. Zhang \\ Department of Pharmacy, The First Affiliated Hospital, Shantou University \\ Medical College, Shantou, China \\ Corresponding author: Y.L. Wang \\ E-mail: YaLiwang1982@163.com
}

Genet. Mol. Res. 14 (3): 10973-10979 (2015)

Received January 29, 2015

Accepted May 14, 2015

Published September 21, 2015

DOI http://dx.doi.org/10.4238/2015.September.21.9

\begin{abstract}
We investigated the effect of flavonoid compounds extracted from species of genus Iris L. on carbon tetrachloride $\left(\mathrm{CCl}_{4}\right)$ induced rat liver fibrosis. Thirty Sprague-Dawley rats were randomly divided into normal control group, liver fibrosis model group, and drug treatment group $\left(\mathrm{N}=10\right.$ each). Next, $0.2 \mathrm{~mL} / 100 \mathrm{~g} \mathrm{CCl}_{4}$ was subcutaneously injected for 6 weeks in both model and treatment rats to generate the liver fibrosis model. In the control group, an equal volume of castor oil was injected subcutaneously. Rats in the treatment group also received $100 \mathrm{mg} \cdot \mathrm{kg}^{-1} \cdot \mathrm{day}^{-1}$ flavonoid compounds via gastric tubes. After 6 weeks, rats were sacrificed, and their liver tissues were examined for pathological changes, including alanine aminotransferase, aspartate aminotransferase, total bilirubin, hyaluronic acid, laminin, and procollagen type-3. Liver tissues from control rats showed no significant pathological changes, while model animals showed significant liver fibrosis. In the treatment group, liver fibrosis significantly decreased compared to the model group $(\mathrm{P}<0.05)$. Liver fibrotic indices, including hyaluronic acid, laminin, and procollagen type-3, in treatment rats were all significantly lower than those in the
\end{abstract}


model group $(\mathrm{P}<0.05)$, but not significantly different compared to the normal group $(\mathrm{P}>0.05)$. Other liver function indices, including alanine aminotransferase, aspartate aminotransferase, and total bilirubin, in treatment rats were also significantly lower than those in model rats $(\mathrm{P}$ $<0.01)$ but higher than those in control animals $(\mathrm{P}<0.05)$. Flavonoid compounds extracted from Iris plants showed significant inhibitory effects on $\mathrm{CCl}_{4}$-induced rat liver fibrosis.

Key words: $\mathrm{CCl}_{4}$; Flavonoid compounds; Liver fibrosis

\section{INTRODUCTION}

Liver fibrosis is a repair mechanism after chronic liver damage and represents an intermediate stage during the progression of chronic liver disease. Without effective management, liver fibrosis often evolves into liver sclerosis or even liver failure (Cengiz et al., 2014; Li et al., 2014a). With timely and effective prevention and treatment, liver fibrosis can be slowed to impede the occurrence and development of liver sclerosis and its complications, thereby reducing pain in patients with chronic liver disease. The pathogenesis of liver fibrosis is complicated, since multiple inducing factors are involved, including the oxidation response, cytokines from different cells, and several growth factors, all of which can induce complicated pathophysiological changes (Kessoku et al., 2014). Although dozens of pathogenic factors exist, the pathological alternations of liver fibrosis are similar: hepatic stellate cell activation is a key step underlying the occurrence of liver fibrosis. The proliferation of hepatic stellate cells can elevate the synthesis and secretion of the extracellular matrix while inhibiting its degradation, causing deposition of extracellular matrix within liver tissues, abnormal hyperplasia of fibrous connective tissues, changes in the structure of hepatic lobules, and finally liver sclerosis. Therefore, the prevention and treatment of liver fibrosis is critical for reversing or inhibiting liver sclerosis. However, the development of effective drugs for reversing liver fibrosis is a major clinical challenge. Recently, promising results in the treatment of liver fibrosis have been reported using Chinese medicine (Li et al., 2014b; Zhang et al., 2014a), which often contain flavonoid compounds (Fu et al., 2014). Current studies have revealed multiple pharmacological roles of flavonoid compounds, including the treatment of tumors, cardiovascular disease, and diabetes (Chavez-Santoscoy et al., 2014; Daddam et al., 2014; Sankari et al., 2014). To further study the effect of flavonoid compounds extracted from Iris plants on liver fibrosis, we developed liver fibrosis model rats using carbon tetrachloride $\left(\mathrm{CCl}_{4}\right)$ and measured indices of liver function, liver fibrosis, and tissue pathological changes to evaluate their effects on $\mathrm{CCl}_{4}$-induced rat liver fibrosis.

\section{MATERIAL AND METHODS}

\section{Research animals}

Rats were used for all experiments, and all procedures were approved by The First Affiliated Hospital of Shantou University Medical College (Guangdong, China).

Thirty healthy male Sprague-Dawley rats aged 2 months (specific pathogen-free grade, body weight $250 \pm 20 \mathrm{~g}$ ) were purchased from the Research Animal Center of Medical Faculty in Shantou University Medical College and were kept in a specific pathogen-free-grade animal center. 


\section{Drugs and reagents}

$\mathrm{CCl}_{4}$ was purchased from Shanghai Chemical Reagent Factory (Shanghai, China) and diluted in castor oil to prepare a $20 \%(\mathrm{v} / \mathrm{v})$ solution. Flavonoid compounds from Iris plants were extracted in the chemical pharmacy laboratory of our university. Major steps in the extraction process included microwave extraction with $70 \%$ ethanol, column filtration using D-101 macroporous resin and polyamide, elution using $70 \%$ ethanol, and vacuum drying. The final product was determined to contain flavonoid compounds by using an $\mathrm{HCl}-\mathrm{Mg}$ qualitative assay. Hematoxylin and eosin were purchased from Boshide Bio. Corp. (Wuhan, China). The radioimmunoassay kits for laminin (LN), hyaluronic acid (HA), and procollagen type-3 (PCIII) were from Kemei Bio. Tech. Corp. (Beijing, China). Alanine aminotransferase (ALT), aspartate aminotransferase (AST), and total bilirubin (TBIL) assay reagents were purchased from Fuxing Changzheng Med. Sci. Corp. (Shanghai, China).

\section{Animal treatment}

Sprague-Dawley rats were randomly divided into 3 groups: normal control group, liver fibrosis model group, and treatment group $(\mathrm{N}=10$ in each group). Liver fibrosis rats were prepared as previously described (Zhang et al., 2014b). $\mathrm{CCl}_{4}$-castor oil solution was subcutaneously injected daily into rats at $0.2 \mathrm{~mL} / 100 \mathrm{~g}$ for 6 weeks. Treatment rats also received flavonoid compounds at $100 \mathrm{mg} \cdot \mathrm{kg}^{-1} \cdot \mathrm{day}^{-1}$ with daily gastric application for 6 weeks in addition to $\mathrm{CCl}_{4}$ treatment in the model rats. In the normal control group, an equal volume of saline was applied daily via gastric tubes for 6 weeks. Food and water were given ad libitum.

\section{Histopathology assays}

After 6 weeks of treatment, all rats were anesthetized using 2\% pentobarbital sodium. Serum was separated from whole blood collected from the abdominal aorta and was stored at $-20^{\circ} \mathrm{C}$. Rats were then sacrificed, and their liver tissues were fixed in $10 \%$ neutral-buffered formaldehyde and stained using hematoxylin and eosin. Liver fibrosis scores were based on the standard recommendation of the Chinese Society of Hepatology (2002) and used to evaluate the severity of liver fibrosis in experimental animals. Briefly, a score ranging from 0-29 was assigned based on the severity of liver fibrosis, with a higher score representing more severe conditions (Qin et al., 2013).

\section{Assays of liver fibrosis and liver function indices}

Serum levels of ALT, AST, TBIL, HA, LN, and PCIII were measured using an automatic biochemical analyzer and radioimmunoassay analyzer.

\section{Statistical analysis}

The SPSS ver. 12.0 software (SPSS, Inc., Chicago, IL, USA) was used to analyze all collected data, which are reported as means \pm SE, unless otherwise specified. Liver fibrosis scores between groups were compared using the rank sum test. Between-group comparisons were conducted using analysis of variance. Statistical significance was defined as $\mathrm{P}<0.05$. 


\section{RESULTS}

\section{Histopathology observations of rat liver tissues}

Liver tissues from all rats were sectioned, stained using hematoxylin and eosin, and observed under a light-field microscope to examine histopathology changes. The results showed no fibrosis in the liver tissues of control rats indicated by: 1) no significant change in liver and hepatic lobular structure and 2) no infiltration of inflammatory cells in liver tissues. The model rats, however, displayed liver fibrosis, including: 1) near the portal area, there were large amounts of collagen fibers, which separated and destructed the hepatic lobular structure; 2) significant alternations in liver tissue and hepatic lobular structure; and 3) numerous infiltrative inflammatory cells developed in the liver tissue, which displayed necrotic hepatocytes and fatty degenerated hepatocytes. After flavonoid treatment, the severity of fibrosis in rats significantly reduced, with fewer fatty degenerated and infiltrative inflammatory hepatocytes. The change in liver fibrosis following treatment was statistically significant compared to model rats $(\mathrm{P}<0.05$; Figure 1 and Table 1), suggesting a significant role of flavonoid compounds extracted from Iris plants in the prevention and treatment of liver fibrosis.
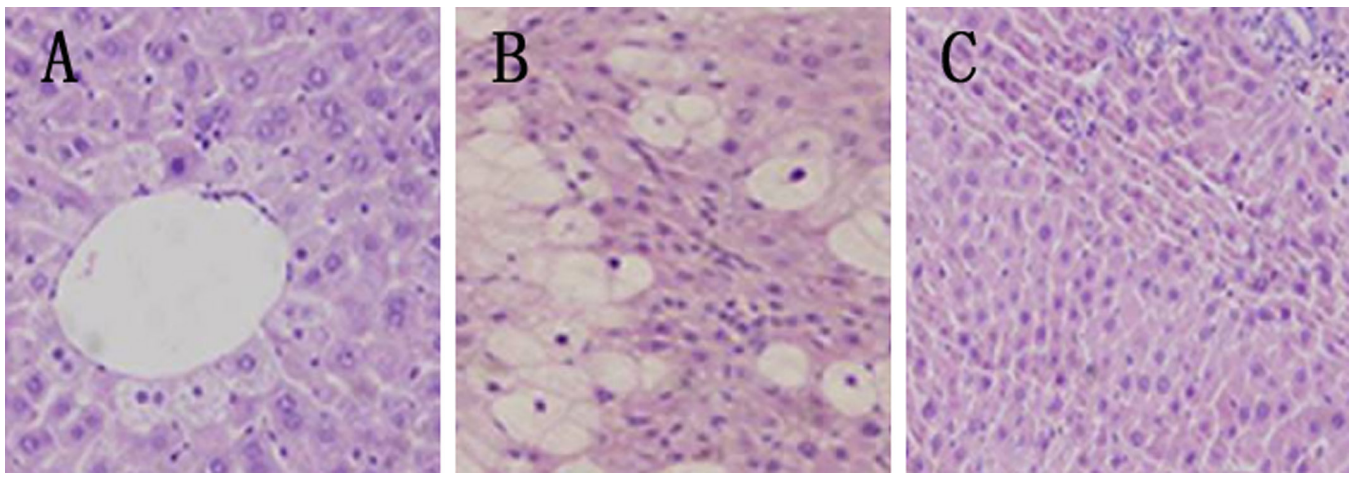

Figure 1. Histopathological changes in rat livers (hematoxylin and eosin staining, 200X). A. Normal control group; B. model group; C. treatment group.

Table 1. Histopathological changes in rat livers.

\begin{tabular}{llc}
\hline Group & $\mathrm{N}$ & Semi-quantitative score of liver fibrosis \\
\hline Control & 10 & $0.81 \pm 0.09$ \\
Model & 10 & $21.42 \pm 3.73^{*}$ \\
Treatment & 10 & $6.71 \pm 1.25^{* \#}$ \\
\hline
\end{tabular}

*P $<0.05$ compared to control group; ${ }^{\#} \mathrm{P}<0.05$ compared to model group.

\section{Indices of liver fibrosis in all groups}

Blood samples were collected from the abdominal aorta in all rats. Serum was separated, and HA, LN, and PCIII levels were measured. The results showed significantly higher serum levels of HA, LN, and PCIII than those in control rats $(\mathrm{P}<0.05$; Table 2$)$. After flavonoid treatment, these indices were found to be significantly lower than those in model group 
rats $(\mathrm{P}<0.05)$, but not statistically different compared to the normal group (Table 2 ), suggesting significant improvement of liver fibrosis following treatment with flavonoid compounds.

Table 2. Indexes of liver fibrosis in all groups.
\begin{tabular}{lcccc}
\hline Group & N & HA & LN & PCIII \\
\hline Control & 10 & $100.21 \pm 11.03$ & $5.23 \pm 0.98$ & $26.11 \pm 2.34$ \\
Model & 10 & $587.43 \pm 32.66^{*}$ & $37.65 \pm 4.15^{*}$ & $89.17 \pm 7.36^{*}$ \\
Treatment & 10 & $106.69 \pm 22.31^{\#}$ & $8.17 \pm 2.18^{*}$ & $38.24 \pm 3.23^{\#}$ \\
\hline
\end{tabular}

$* \mathrm{P}<0.05$ compared to control group; ${ }^{*} \mathrm{P}<0.05$ compared to model group.

\section{Liver function indices in all rats}

Blood samples were collected from the abdominal aorta of rats in all groups. Serum was separated and tested for the levels of ALT, AST, and TBIL, which indicate hepatic functions. The result showed significantly elevated ALT, AST, and TBIL levels in model rats compared to control rats $(\mathrm{P}<0.05$; Table 3$)$. After flavonoid treatment, these indices significantly decreased compared to the model group $(\mathrm{P}<0.05)$, but remained significantly higher than those in control rats $(\mathrm{P}<0.05$; Table 3$)$.

Table 3. Levels of ALT, AST, and TBIL in all groups.

\begin{tabular}{llcll}
\hline Group & $\mathrm{N}$ & ALT $(\mathrm{IU} / \mathrm{L})$ & AST $(\mathrm{IU} / \mathrm{L})$ & TBIL $(\mu \mathrm{M})$ \\
\hline Control & 10 & $37.35 \pm 4.16$ & $225.89 \pm 41.62$ & $1.26 \pm 0.21$ \\
Model & 10 & $438.43 \pm 52.16^{*}$ & $667.37 \pm 78.68^{*}$ & $4.27 \pm 0.56^{*}$ \\
Treatment & 10 & $236.41 \pm 67.18^{* \#}$ & $395.13 \pm 92.68^{* \#}$ & $2.98 \pm 0.67^{* \#}$ \\
\hline
\end{tabular}

*P $<0.05$ compared to control group; ${ }^{*} \mathrm{P}<0.05$ compared to model group.

\section{DISCUSSION}

Because of environmental factors and changes in eating habits and lifestyles, the global occurrence rate of hepatic disease has increased in recent years. Liver sclerosis is a severe complication that impairs the quality of life of patients with hepatic disease. Chronic liver damage or liver sclerosis can be caused by multiple factors, including drug abuse, toxicants, viral infection, and ethanol toxicity, but in China, it often occurs after hepatitis is acquired (Mokdad et al., 2014; Xu et al., 2014). Recent studies have found that liver fibrosis occurs during the pathogenesis of liver sclerosis, which is chronic advancing hepatic damage. Therefore, the effective prevention and treatment of liver fibrosis is important for impeding or even reversing the development of liver sclerosis. However, few medications are available that target liver fibrosis, since the basic mechanism is not well understood. Pharmacological research of Chinese medicine has shown great advancements in recent years. These medicines have been shown to have an anti-fibrotic effect in animal studies and preliminary clinical trials (Chien et al., 2014; Wang et al., 2014).

The pharmacological functions of flavonoid compounds include multiple-treatment effects against various systematic diseases, including cardiovascular, neurological, immunological, endocrinology, and respiratory symptoms (Chavez-Santoscoy et al., 2014; Daddam et al., 2014; Sankari et al., 2014). These studies also revealed promising treatment effects 
of flavonoid compounds against liver damage. In this study, we measured the function of flavonoid compounds extracted from Iris plants for preventing and treating liver fibrosis in a $\mathrm{CCl}_{4}$-induced fibrosis rat model. The result showed significantly lower severity of fibrosis after flavonoid compound treatment, suggesting a role of flavonoid compounds from Iris plants in preventing and treating liver fibrosis. Further assays of indices of liver fibrosis showed that HA, LN, and PCIII levels after treatment significantly decreased, illustrating that flavonoid compounds from Iris plants can improve liver fibrosis. Furthermore, liver function indices, including ALT, AST, and TBIL, were improved after flavonoid treatment, but remained significantly high compared to control animals, suggesting that flavonoids can function as a replenishment method in ameliorating liver damage and significantly preventing liver fibrosis. Studies have shown that flavonoid compounds can protect the liver through multiple pathways, such as by preventing high liver iron levels, inhibiting lipid peroxidation and hepatic protein oxidation, and facilitating iron excretion (Feher and Lengyel, 2012; Sak, 2014). Additionally, flavonoid compounds have a stronger anti-oxidative activity than vitamin $\mathrm{C}$ and vitamin E (Mehta et al., 2013). In contrast, flavonoid compounds can exert their protective role by regulating cell apoptosis; these compounds can inhibit normal hepatic cell apoptosis while accelerating apoptosis of tumor cells and necrotic hepatic cells. Flavonoid compounds also protect liver against damage via modulation of cell mitosis and proliferation as well as the secretion of enzymes against platelets during coagulation and inflammation (Dong et al., 2013). Some research suggests that lipopolysaccharide can induce hepatic cells to produce tumor necrosis factor, which can further induce the inflammatory response to accelerate the development of liver sclerosis. Flavonoid compounds can inhibit tumor necrosis factor secretion to effectively impede liver damage (Park et al., 2013). The detailed cellular mechanism of flavonoid compounds extracted from Iris plants, however, should be further examined.

In summary, we demonstrated the potent anti-fibrotic role of flavonoid compounds extracted from Iris plants in animal experiments, providing a theoretical basis for its clinical application and revealing an alternative method for the clinical treatment of liver fibrosis.

\section{Conflicts of interest}

The authors declare no conflict of interest.

\section{REFERENCES}

Cengiz M, Ozenirler S, Yücel AA and Yilmaz G (2014). Can serum pin1 level be regarded as an indicative marker of nonalcoholic steatohepatitis and fibrotic stages? Digestion 90: 35-41.

Chavez-Santoscoy RA, Gutierrez-Uribe JA, Granados O, Torre-Villalvazo I, et al. (2014). Flavonoids and saponins extracted from black bean (Phaseolus vulgaris L.) seed coats modulate lipid metabolism and biliary cholesterol secretion in C57BL/6 mice. Br. J. Nutr. 112: 886-899.

Chien SC, Chang WC, Lin PH, Chang WP, et al. (2014). A Chinese herbal medicine, jia-wei-xiao-yao-san, prevents dimethylnitrosamine-induced hepatic fibrosis in rats. Sci.World J. 2014: 217525.

Daddam JR, Dowlathabad MR, Panthangi S and Jasti P (2014). Molecular docking and P-glycoprotein inhibitory activity of flavonoids. Interdiscip. Sci. 6: 167-175.

Dong M, Hong T, Liu S, Zhao J, et al. (2013). Hepatoprotective effect of the flavonoid fraction isolated from the flower of Inula britannica against D-galactosamine-induced hepatic injury. Mol. Med. Rep. 7: 1919-1923.

Feher J and Lengyel G (2012). Silymarin in the prevention and treatment of liver diseases and primary liver cancer. Curr. Pharm. Biotechnol. 13: 210-217.

Fu X, Zhang J, Guo L, Xu Y, et al. (2014). Protective role of luteolin against cognitive dysfunction induced by chronic cerebral hypoperfusion in rats. Pharmacol. Biochem. Behav. 126: 122-130. 
Kessoku T, Ogawa Y, Yoneda M, Imajo K, et al. (2014). Simple scoring system for predicting cirrhosis in nonalcoholic fatty liver disease. World J. Gastroenterol. 20: 10108-10114.

Li JF, Liu S, Ren F, Liu M, et al. (2014a). Fibrosis progression in interferon treatment-naive Chinese plasma donors with chronic hepatitis C for 20 years: a cohort study. Int. J. Infect. Dis. 27: 49-53.

Li W, Wu Y, Zhu C, Wang Z, et al. (2014b). Anti-fibrosis effects of Huisheng oral solution in CCl4-induced hepatic fibrosis in rat. Indian J. Pharmacol. 46: 216-221.

Mehta RR, Katta H, Kalra A, Patel R, et al. (2013). Efficacy and mechanism of action of Deguelin in suppressing metastasis of $4 \mathrm{~T} 1$ cells. Clin. Exp. Metastasis 30: 855-866.

Mokdad AA, Lopez AD, Shahraz S, Lozano R, et al. (2014). Liver cirrhosis mortality in 187 countries between 1980 and 2010: a systematic analysis. BMC Med. 12: 145.

Park HY, Choi HD, Eom H and Choi I (2013). Enzymatic modification enhances the protective activity of citrus flavonoids against alcohol-induced liver disease. Food Chem. 139: 231-240.

Qin D, Wen Z, Nie Y and Yao G (2013). Effect of Cichorium glandulosum extracts on CCl4-induced hepatic fibrosis. Iran Red Crescent. Med. J. 15: e10908.

Sak K (2014). Cytotoxicity of dietary flavonoids on different human cancer types. Pharmacogn. Rev. 8: 122-146.

Sankari SL, Babu NA, Rani V, Priyadharsini C, et al. (2014). Flavonoids - clinical effects and applications in dentistry: a review. J. Pharm. Bioallied Sci. 6: S26-S29.

Wang ZC, Yang S, Huang JJ, Chen SL, et al. (2014). Effect of Rougan Huaqian granules combined with human mesenchymal stem cell transplantation on liver fibrosis in cirrhosis rats. Asian Pac. J. Trop. Med. 7: 576-581.

Xu B, Lin L, Xu G, Zhuang Y, et al. (2014). Long-term lamivudine treatment achieves regression of advanced liver fibrosis/cirrhosis in patients with chronic hepatitis B. J. Gastroenterol. Hepatol. Epub ahead of print.

Zhang LJ, Sun MY, Ning BB, Zhang WM, et al. (2014a). Xiayuxue Decoction ([symbols; see text]) attenuates hepatic stellate cell activation and sinusoidal endothelium defenestration in CCl4-induced fibrotic liver of mice. Chin. J. Integr. Med. 20: 516-523.

Zhang Y, Chen XM and Sun DL (2014b). Effects of coencapsulation of hepatocytes with adipose-derived stem cells in the treatment of rats with acute-on-chronic liver failure. Int. J. Artif. Organs. 37: 133-141. 